\title{
Co-existing inflammatory bowel disease and Barrett's esophagus is associated with esophageal dysplasia: a propensity score-matched cohort
}

\section{다 (1) $(-)$}

Authors

Matthew Fasullo ${ }^{1}$, Aditya Sreenivasen², Erik Holzwanger ${ }^{3}$, Charles Lavender ${ }^{4}$, Milan Patel ${ }^{1}$, Tilak Shah ${ }^{1}$, Pritesh Mutha $^{1}$, Robert F. Yacavone ${ }^{3}$, Keith Sultan ${ }^{2}$, Arvind J. Trindade ${ }^{2}$, George Smallfield ${ }^{1}$

Institutions

1 Division of Gastroenterology, Hepatology, and Nutrition, Virginia Commonwealth University Medical Center, Richmond, Virginia, United States

2 Division of Gastroenterology, Zucker School of Medicine at Hofstra/Northwell, Long Island Jewish Medical Center, New Hyde Park, New York, United States

3 Division of Gastroenterology \& Hepatology, Tufts Medical Center, Boston, Massachusetts, United States

4 Department of Internal Medicine, University of Arkansas for Medical Sciences, Little Rock, Arkansas, United States

submitted 12.2.2021

accepted after revision 27.5.2021

\section{Bibliography}

Endosc Int Open 2021; 09: E1524-E1529

DOI 10.1055/a-1526-0507

ISSN 2364-3722

(c) 2021. The Author(s).

This is an open access article published by Thieme under the terms of the Creative Commons Attribution-NonDerivative-NonCommercial License, permitting copying and reproduction so long as the original work is given appropriate credit. Contents may not be used for commercial purposes, or adapted, remixed, transformed or built upon. (https://creativecommons.org/licenses/by-nc-nd/4.0/)

Georg Thieme Verlag KG, Rüdigerstraße 14,

70469 Stuttgart, Germany

Corresponding author

Matthew Fasullo, Virginia Commonwealth University Medical Center - Gastroenterology, 1250 E Marshall Street, Richmond, VA 23298, United States

Fax: +1-804-828-3673

matthewj.fasullo@gmail.com

\section{ABSTRACT}

Background and study aims Barrett's esophagus (BE) and inflammatory bowel disease (IBD) predispose to the development of dysplasia and cancer. It is unclear if the inflammatory cascade seen in IBD affects disease progression in BE. We aimed to determine if patients with BE who have co-existing IBD had a higher risk of dysplasia, nodular disease, or longer segments than BE patients without IBD.

Patients and methods This was a multicenter, retrospective propensity score-matched cohort study. We compared rates of dysplasia, nodular disease, and segment length in patients with BE and IBD (cases) to patients with BE who did not have IBD (controls). Controls were 1:1 propensity score matched with controls for age, sex, body mass index (BMI), smoking, and hiatal hernia.

Results A total of 132 patients were included in the IBD + BE group and 132 patients in the BE group. Patients with $\mathrm{IBD}+\mathrm{BE}$ had higher rates of esophageal dysplasia compared to controls ( $15.9 \%$ vs. $6.1 \%$ [adjusted odds ratio [OR]: 2.9 , $95 \% \mathrm{Cl}: 1.2-6.9$ ]) and more nodules (9.8\% vs. $3.0 \%$ [adjusted OR: $3.5,95 \% \mathrm{Cl}: 1.1-11.0])$. IBD + BE group was also associated with longer $B E$ segments (43.9\% vs. $12.1 \%$ [OR: 5.7, $95 \% \mathrm{Cl}: 3.0-10.6])$.

Conclusions Co-existing IBD may increase the risk of dysplasia and esophageal nodules in patients with BE. Our findings may have implications for BE surveillance intervals in IBD patients. Prospective studies are needed to confirm

\section{Introduction}

Barrett's esophagus (BE) is a condition in which normal stratified squamous epithelium of the distal esophagus is replaced by metaplastic columnar epithelium [1]. BE prevalence in the
United States ranges from $0.5 \%$ to $2 \%$ [2]. BE carries a risk of progressing to esophageal adenocarcinoma (EAC), but the overall magnitude of this risk is low $(<1 \%)$ in patients without dysplasia [3]. Risk of adenocarcinoma increases in the setting of dysplasia. Low-grade dysplasia (LGD) carries a $0.2 \%$ to $1.2 \%$ 
annual risk and high-grade dysplasia (HGD) has an annual risk of $4 \%$ to $8 \%$ of progression to EAC $[4,5]$.

Several studies have assessed risk factors for progression of BE to dysplasia and EAC. Risk factors have been those that predispose to $B E$, such as longstanding gastroesophageal reflux disease (GERD), hiatal hernia, older age, obesity, and smoking [6]. Our group anecdotally noted that patients with BE who have IBD seemed to have higher rates of dysplasia than patients with BE who did not have IBD. We hypothesized that IBD may increase the risk of dysplasia and EAC in BE via several potential mechanisms, including upregulation of inflammatory cytokines [7], changes to bile acids [8,9], and perturbations in the microbiome $[10,11]$.

The aim of the current study was to assess whether these findings persist when assessed in a larger sample of IBD and BE patients across multiple centers in the United States, when compared to a propensity score-matched group of controls with BE who did not have IBD.

\section{Patients and methods}

This was a retrospective study conducted at four tertiary care academic medical centers in the United States (Virginia Commonwealth University Medical Center, Zucker School of Medicine at Hofstra/Northwell, University of Arkansas Medical Center, and Tufts University Medical Center). The institutional review boards at all participating centers approved this study.

\section{Patient selection}

At all four centers, we queried the medical records database with the ICD-10 code k22.7 to identify patients diagnosed with BE between 2009 and 2019. We reviewed the medical records to assess whether patients met inclusion criteria. Patients were included if they were $\geq 18$ years of age with a diagnosis of $B E$. Patients with BE were excluded from the study if biopsy results did not confirm intestinal metaplasia in the esophagus. Patients were excluded if they had endoscopic or histopathologic evidence of esophagitis. Among patients identified as having a diagnosis of $\mathrm{BE}$, we queried the medical records database with ICD-10 codes K50* for Crohn's disease (CD) and $\mathrm{K} 51^{*}$ for ulcerative colitis (UC). Patients were excluded from the IBD arm if they did not have an endoscopic biopsy confirming presence of CD or UC and if they had a diagnosis of indeterminate colitis. For patients who the met inclusion criteria, we abstracted information on demographics, endoscopy findings, pathology results, and concomitant IBD therapy. Patients with BE and IBD $(I B D+B E)$ were considered the active study arm. In the BE without IBD group (controls), we performed 1:1 propensity score matching using a maximum caliper adjustment of $<0.25$ using nearest-neighbor matching. Cases were matched with controls for the following variables potentially associated with an increased risk of dysplasia in the literature: age, sex, presence of a hiatal hernia, body mass index (BMI), and history/use of tobacco.

\section{Endoscopy procedures}

The typical protocol for BE surveillance at all institutions was to perform careful high-definition white light examination (HDWLE) followed by random biopsies in four quadrants at $1-$ to 2-cm intervals (i. e. - Seattle protocol biopsies). At all four centers that participated in the study, BE samples are interpreted by gastrointestinal pathologists with expertise in $\mathrm{BE}$ and a finding of dysplasia is confirmed by a second pathologist. If an area of nodularity was identified on HD-WLE, then endoscopic mucosal resection was performed to better assess (and potentially treat) that area for dysplasia or cancer before performing Seattle protocol biopsies of the remaining BE segment. Electronic chromoendoscopy was performed at the discretion of the attending gastroenterologist. No other tools to detect dysplasia were used, including distal attachment caps and acetic acid.

\section{Statistical analysis}

All statistical analyses were performed using SPSS v. 26 (SPSS Inc., Chicago, Illinois, United States). Baseline variables in each group were reported as mean \pm standard deviation (SD) for continuous variables, and frequencies and percentages for categorical variables. We compared baseline variables in the two groups using Student's t-test for normally distributed continuous variables, and Fisher's exact test for categorical variables. The primary outcome was the rate of esophageal dysplasia in cases $(B E+I B D)$ versus controls (BE only). Secondary outcomes were $B E$ segment length and rate of nodular disease between cases and controls. The primary outcome was measured using Fisher's exact test. $P<0.05$ was considered statistically significant. Separate multivariate logistic regression analyses were performed to control for confounding risks factors for dysplasia and nodules.

\section{Results}

We queried the medical records database of 648 patients. A total of 384 patients were excluded from our study for whom we could not confirm an adequate histopathologic diagnosis of either BE or IBD. A total of 264 patients were included (132 $\mathrm{IBD}+\mathrm{BE}$ matched to 132 controls). Baseline demographics are listed in > Table 1. The majority of patients in both groups were white, male, and overweight. In the IBD + BE group, 72 (54.5\%) had CD while 60 patients (45.6\%) had UC. Among patients with IBD, 36 (27.3\%) were not on any disease-specific therapy, while 61 (46.2\%) were on 5-aminosalicyclic acid derivatives, 18 (13.6\%) were on biologic therapy, and 17 (12.9\%) were on immunomodulators (azathioprine or methotrexate).

\section{Primary and secondary outcomes}

The percentage of patients who had any degree of dysplasia was $10.9 \%$ ( $\triangleright$ Table 2 ). Patients in the IBD + BE group had a higher rate of any dysplasia formation compared to the $\mathrm{BE}$ group (21 [15.9\%] vs. 8 [6.1\%], $P<0.01)$. In particular, they had significantly higher rates of LGD compared to the BE group (12 [12.1\%] vs. 4 [3.0\%], $P<0.01)$. Patients in the IBD + BE group had a similar rate of HGD compared to the BE group 
- Table 1 Patient characteristics.

\begin{tabular}{|c|c|c|c|}
\hline Variables & $\begin{array}{l}\text { IBD + BE } \\
(n=132)\end{array}$ & $\begin{array}{l}\text { BE } \\
(n=132)\end{array}$ & P value \\
\hline 1 Race, White, n (\%) & $115(87.1 \%)$ & $113(85.6 \%)$ & 0.58 \\
\hline Sex, male, n (\%) & $89(67.4 \%)$ & $90(68.2 \%)$ & NA \\
\hline BMI $\left(\mathrm{kg} / \mathrm{m}^{2}\right)$, mean & 29.0 & 29.1 & NA \\
\hline Charlson Cl (SD) & $3.2(2.1)$ & $3.0(2.1)$ & 0.54 \\
\hline Age, mean (SD) & $57.5(12.1)$ & $57.1(14.9)$ & 0.79 \\
\hline Hiatal hernia, n (\%) & $92(69.7 \%)$ & $91(68.9 \%)$ & NA \\
\hline Tobacco use, n (\%) & $76(57.6 \%)$ & $76(57.6 \%)$ & NA \\
\hline Alcohol use, n (\%) & $88(66.7 \%)$ & $73(55.3 \%)$ & 0.06 \\
\hline PPI use, n (\%) & $122(92.4 \%)$ & $114(86.4 \%)$ & 0.11 \\
\hline Aspirin use, n (\%) & $46(34.8 \%)$ & $33(25.0 \%)$ & 0.08 \\
\hline NSAIDs use, n (\%) & $9(6.8 \%)$ & $15(11.4 \%)$ & 0.20 \\
\hline Crohn's disease, n (\%) & $72(54.5 \%)$ & & \\
\hline Immunomodulator, n (\%) & $17(12.9)$ & & \\
\hline Biologics, n (\%) & $18(13.6)$ & & \\
\hline 5-ASA, n (\%) & $61(46.2)$ & & \\
\hline No therapy, n (\%) & $36(27.3)$ & & \\
\hline \multicolumn{4}{|c|}{$\begin{array}{l}\text { IBD, inflammatory bowel disease; BE, Barrett's esophagus; CI, comorbidity } \\
\text { index; SD, standard deviation; NA, not applicable; PPI, proton pump inhibi- } \\
\text { tor; NSAID, nonsteroidal anti-inflammatory drug; 5-ASA, 5-aminosalicylic } \\
\text { acid. }\end{array}$} \\
\hline
\end{tabular}

(5 [3.8\%] vs. 4 [3.0\%], $P=0.73$ ). Patients in the IBD + BE group had a similar rate of adenocarcinoma compared to the $\mathrm{BE}$ group (7 [5.3\%] vs. $6[4.6 \%], P=0.78)$. Patients in the IBD + BE group had a higher rate of nodule formation compared to the $\mathrm{BE}$ group (13 [9.8\%] vs. 4 [3.0\%], $P=0.02$ ). Finally, patients with $\mathrm{IBD}+\mathrm{BE}$ had longer $\mathrm{BE}$ segment lengths compared to the $\mathrm{BE}$ group (58 [43.9\%] vs. 16 [12.1\%], $P<0.01)(\triangleright$ Table 2). On univariate regression, IBD was the only independent variable associated with dysplasia, OR: $2.9,95 \% \mathrm{Cl}: 1.0-6.2, P=0.05$. On multivariable regression, IBD was the only independent variable associated with dysplasia or nodule formation, OR: $2.9,95 \% \mathrm{Cl}$ 1.3-6.9, $P=0.01$ and $\mathrm{OR}: 3.5,95 \% \mathrm{Cl}: 1.1-11.0, P=0.03$, respectively. Neither long segment $B E$, alcohol use, nor Charlson comorbidity index were associated with a statistically significant risk for these outcomes ( $\downarrow$ Table 3 ). Accounting for Barret's segment length, a separate analysis demonstrated that the prevalence of dysplasia was similar between the IBD+BE and BE group in those who has long segment BE ( $\vee$ Table 4$)$. Subgroup analyses performed for UC and CD separately were similar between groups and are reported in table/supplementary material (Supplementary Table 1 and Table 2).

\section{Discussion}

In this multicenter matched case-control study, we revealed that patients with IBD who have BE have a significantly higher point prevalence of dysplasia and nodular disease, when compared to matched BE patients without IBD. This increased risk was seen with both UC and CD. Intestinal metaplasia is thought to arise as a response to chronic mucosal inflammation and injury of the esophagus, primarily from GERD [12]. IBD patients often have evidence of systemic inflammation with an increase in multiple pro-inflammatory cascades. If the pathogenesis of increased BE severity is related primarily to inflammation, then other inflammatory conditions should theoretically predispose to a more severe BE phenotype. However, the systemic pro-inflammatory state in IBD does not necessarily result in histologic evidence of esophageal mucosal inflammation and thus, other mechanisms may be involved $[13,14]$.

This is the first study to demonstrate an association between IBD and a more severe BE phenotype. The data were collected from a relatively large cohort of patients across multiple centers in the United States, and propensity score matching was utilized to identify an appropriate group for comparison. Prior studies have demonstrated that long-segment BE is associated with increased risk for dysplasia and increased propensity to progress to esophageal adenocarcinoma [15]. Including only patients with long-segment $\mathrm{BE}$, we again demonstrated that the presence of IBD was associated with a greater risk for dys-

> Table 2 Primary outcomes, IBD + BE vs. BE.

\begin{tabular}{|c|c|c|c|c|}
\hline Variables & $\begin{array}{l}\text { IBD + BE, } n(\%) \\
(n=132)\end{array}$ & $\begin{array}{l}\text { BE, } n(\%) \\
(n=132)\end{array}$ & OR $(95 \% \mathrm{Cl})$ & $P$ value \\
\hline Any dysplasia & $21(15.9 \%)$ & $8(6.1 \%)$ & $2.9(1.2-6.9)$ & $<0.01^{1}$ \\
\hline LGD & $16(12.1 \%)$ & $4(3.0 \%)$ & $4.4(1.4-13.6)$ & $<0.01^{1}$ \\
\hline HGD & $5(3.8 \%)$ & $4(3.0 \%)$ & $1.3(0.3-4.8)$ & 0.73 \\
\hline Adenocarcinoma & $7(5.3 \%)$ & $6(4.6 \%)$ & $1.2(0.4-3.6)$ & 0.78 \\
\hline Nodule & $13(9.8 \%)$ & $4(3.0 \%)$ & $3.4(1.1-11.0)$ & $0.02^{2}$ \\
\hline Long Barrett's esophagus & $58(43.9 \%)$ & $16(12.1 \%)$ & $5.7(3.0-10.6)$ & $<0.01^{1}$ \\
\hline
\end{tabular}


- Table 3 Binary logistic regression models.

\begin{tabular}{|c|c|c|c|c|c|}
\hline Model & Variables & $\begin{array}{l}\text { Univariate } \\
\text { (OR, } 95 \% \mathrm{Cl})\end{array}$ & $P$ value & $\begin{array}{l}\text { Multivariate } \\
\text { (OR, } 95 \% \mathrm{CI})\end{array}$ & $P$ value \\
\hline \multirow[t]{4}{*}{ Model 1: dysplasia } & Alcohol & $0.5(0.2-1.3)$ & 0.16 & - & -- \\
\hline & Long-segment BE & $0.8(0.3-1.8)$ & 0.52 & - & - \\
\hline & Charlson Cl & $1.0(0.8-1.2)$ & 0.69 & - & - \\
\hline & $\mathrm{IBD}$ & $2.9(1.0-6.2)$ & $0.05^{1}$ & $2.9(1.3-6.9)$ & $0.01^{1}$ \\
\hline \multirow[t]{4}{*}{ Model 2: nodules } & Alcohol & $0.7(0.2-2.1)$ & 0.54 & - & - \\
\hline & Long-segment BE & $0.7(0.3-2.2)$ & 0.59 & - & - \\
\hline & Charlson $\mathrm{Cl}$ & $0.9(0.7-1.2)$ & 0.45 & - & - \\
\hline & $\mathrm{IBD}$ & $3.4(0.9-10.5)$ & 0.06 & $3.5(1.1-11.0)$ & $0.03^{1}$ \\
\hline
\end{tabular}

- Table4 Dysplasia risks associated with long-segment Barrett's esophagus, IBD + BE vs. BE.

\begin{tabular}{|c|c|c|c|c|}
\hline Variables & $\begin{array}{l}\text { IBD + BE, n (\%) } \\
(n=58)\end{array}$ & $\begin{array}{l}B E, n(\%) \\
(n=17)\end{array}$ & OR $(95 \% \mathrm{Cl})$ & $P$ value \\
\hline Any dysplasia & 12 (20.7\%) & $1(5.9 \%)$ & $4.2(0.5-34.7)$ & 0.12 \\
\hline LGD & $10(17.2 \%)$ & $1(5.9 \%)$ & $3.3(0.4-28.1)$ & 0.21 \\
\hline HGD & $2(3.4 \%)$ & 0 & $1.0(0.9-1.1)$ & 0.31 \\
\hline Adenocarcinoma & $3(5.2 \%)$ & $1(5.9 \%)$ & $0.9(0.1-9.0)$ & 0.91 \\
\hline
\end{tabular}

plasia, indicating another mechanism likely accounts for these findings. Although this is the first study of its kind to show an association between the presence of IBD and dysplasia risk in patients with BE, we have several hypotheses to help explain these findings, including luminal dysbiosis, changes to bile acids, and alteration in gene expression. Whether IBD predisposes to oral dysbiosis along with alterations in bile acids and their impact on altering gene expression in esophageal epithelium warrants further investigation.

While this observation is thought-provoking, our results should be interpreted in the context of certain limitations. IBD and BE patients were identified using ICD-10 codes. To address this limitation, we did review the medical record to confirm the accuracy of both BE and IBD diagnoses. Our primary endpoint was the point prevalence of LGD, a diagnosis with considerable disagreement between pathologists. Interestingly, the prevalence of HGD and adenocarcinoma failed to reach statistical significance likely reflecting too small a sample size to detect this difference. Although we identified a strong association between BE severity and IBD, our study was not designed to address the effect of IBD severity and the effect of various IBD therapies on study outcomes. Another limitation is that the number of endoscopies and length of follow up in each group cannot be ascertained from each cohort due the majority of patients being referred to a tertiary care center for management of their BE. Finally, as with any observational study, we recognize that correlation does not imply causation.

\section{Conclusions}

In summary, this is the first study to document an increased risk of dysplasia in BE patients who have co-existing IBD. Our findings may have implications for surveillance intervals in this subgroup of BE patients. Our results could inform risk-benefit discussions with BE in IBD patients who are considering further endoscopic interventions. We believe more studies are needed to confirm these findings, possibly at a population level. Further investigation is also warranted to assess whether other inflammatory conditions predispose to increased BE severity along with translational studies to better understand why IBD may predispose to a more severe BE phenotype. 


\section{Competing interests}

Dr. Shah is on the research advisory board for CSA Medical and receives research support from Allergan and Lucid. Dr. Mutha receives research support from Allergan and Lucid. Dr. Trindade is a consultant to Pentax Medical and Olympus American and receives research support from Ninepoint Medical. Dr. Smallfield receives research support from and is on the research advisory board for CSA medical and receives research support from Pentax/C2 Therapeutics and Lucid.

\section{References}

[1] Hayeck T], Kong CY, Spechler S] et al. The prevalence of Barrett's esophagus in the US: estimates from a simulation model confirmed by SEER data. Dis Esophagus 2010; 23: 451-457

[2] Runge TM, Abrams JA, Shaheen NJ. Epidemiology of Barrett's esophagus and esophageal adenocarcinoma. Gastroenterol Clin North Am 2015; 44: 203-231

[3] Spechler S]. Barrett's esophagus. Semin Gastrointest Dis 1996; 7: 5160

[4] Spechler SJ. Disputing dysplasia. Gastroenterology 2001; 120: 18641868

[5] Kestens C, Leenders M, Offerhaus G] et al. Risk of neoplastic progression in Barrett's esophagus diagnosed as indefinite for dysplasia: a nationwide cohort study. Endoscopy 2015; 47: 409-414
[6] Orloff M, Peterson C, He X et al. Germline mutations in msr1, ascc1, and cthrc 1 in patients with barrett esophagus and esophageal adenocarcinoma. JAMA 2011; 306: 410-419

[7] Zhang HY, Hormi-Carver K, Zhang X et al. In benign Barrett's epithelial cells, acid exposure generates reactive oxygen species that cause DNA double-strand breaks. Cancer Res 2009; 69: 9083-9089

[8] Rohr M, Aljabban J, Rudeski-Rohr T et al. Meta-analysis reveals the prognostic relevance of nuclear and membrane-associated bile acid receptors in gastric cancer. Clin Transl Gastroenterol 2021; 12: e00295

[9] Sun D, Wang X, Gai Z et al. Bile acids but not acidic acids induce Barrett's esophagus. Int J Clin Exp Pathol 2015; 8: 1384-1392

[10] Snider E], Compres G, Freedberg DE et al. Barrett's esophagus is associated with a distinct oral microbiome. Clin Transl Gastroenterol 2018; 9: 135

[11] Okereke IC, Miller AL, Jupiter DC et al. Microbiota detection patterns correlate with presence and severity of Barrett's esophagus. Front Cell Infect Microbiol 2021; 11: 555072

[12] Phillips WA, Lord RV, Nancarrow D] et al. Barrett's esophagus. J Gastroenterol Hepatol 2011; 26: 639-648

[13] Turner D, Griffiths AM. Esophageal, gastric, and duodenal manifestations of IBD and the role of upper endoscopy in IBD diagnosis. Curr Gastroenterol Rep 2007; 9: 475-478

[14] Pimentel AM, Rocha R, Santana GO. Crohn's disease of esophagus, stomach and duodenum. World J Gastrointest Pharmacol Ther 2019; 10: $35-49$

[15] Spechler SJ. Barrett esophagus and risk of esophageal cancer: a clinical review. JAMA 2013; 310: 627-636 
Supplementary Table 1 Primary outcomes for UC+BE vs. BE.

\begin{tabular}{|c|c|c|c|c|}
\hline Variables & $\begin{array}{l}U C+B E, n(\%) \\
(n=60)\end{array}$ & $\begin{array}{l}\text { BE, } n(\%) \\
(n=114)\end{array}$ & OR $(95 \% \mathrm{CI})$ & $P$ value \\
\hline Any dysplasia & $10(16.7 \%)$ & $8(6.1 \%)$ & $3.10(1.2-8.3)$ & $0.03^{1}$ \\
\hline LGD & $8(13.3 \%)$ & $4(3.0 \%)$ & $4.92(1.4-17.1)$ & $0.01^{1}$ \\
\hline HGD & $2(3.3 \%)$ & $4(3.0 \%)$ & $1.10(0.2-6.2)$ & 0.91 \\
\hline Adenocarcinoma & $3(5.0 \%)$ & $6(4.6 \%)$ & $1.10(0.3-4.6)$ & 0.89 \\
\hline Nodule & $6(10.0 \%)$ & $4(3.0 \%)$ & $3.55(1.0-13.1)$ & $0.05^{1}$ \\
\hline Long Barrett's esophagus & $26(43.3 \%)$ & $16(12.1 \%)$ & $5.54(2.7-11.5)$ & $<0.01^{2}$ \\
\hline
\end{tabular}

- Supplementary Table 2 Primary outcomes for CD + BE vs. BE.

\begin{tabular}{|c|c|c|c|c|}
\hline Variables & $\begin{array}{l}C D+B E, n(\%) \\
(n=72)\end{array}$ & $\begin{array}{l}B E, n(\%) \\
(n=132)\end{array}$ & OR $(95 \% \mathrm{CI})$ & $P$ value \\
\hline Any Dysplasia & $11(15.3 \%)$ & $8(6.1 \%)$ & $2.79(1.1-7.3)$ & $0.04^{1}$ \\
\hline LGD & $8(11.1 \%)$ & $4(3.0 \%)$ & $4.00(1.2-13.7)$ & $0.02^{1}$ \\
\hline HGD & $3(4.2 \%)$ & $4(3.0 \%)$ & $1.39(0.3-6.4)$ & 0.67 \\
\hline Adenocarcinoma & $4(5.6 \%)$ & $6(4.6 \%)$ & $1.24(0.3-4.5)$ & 0.75 \\
\hline Nodule & $7(9.7 \%)$ & $4(3.0 \%)$ & $3.45(1.0-12.2)$ & $0.05^{1}$ \\
\hline Long Barrett's esophagus & $32(44.4 \%)$ & $16(12.1 \%)$ & $5.80(2.9-11.7)$ & $<0.01^{2}$ \\
\hline \multicolumn{5}{|c|}{$\begin{array}{l}\text { CD, Crohn's disease; BE, Barrett's esophagus; OR, odds ratio; CI, confidence interval; LGD, low-grade dysplasia; HGD, high-grade dysplasia. } \\
1 P<0.05 \text {. } \\
2 P<0.01\end{array}$} \\
\hline
\end{tabular}

